\title{
Progress on neglected disease is moot if we neglect to count
}

\author{
Donald R Hopkins
}

\begin{abstract}
The recent global campaign launched against a select number of neglected tropical diseases (NTDs) is a welcome development. But we should be as careful about measuring progress toward the control or elimination of these diseases as we are about choosing which ones to target.
\end{abstract}

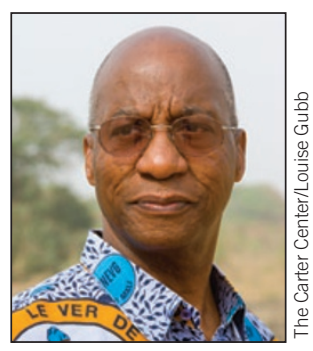

The World Health Organization (WHO) has done the world a great service by raising awareness of the need and opportunity to reduce poverty by addressing certain NTDs that are associated with poverty. In October, it issued its first report on the extent of NTDs and set out a plan for collective action against 17 of them, from Chagas disease to leprosy to Guinea worm disease (http://www.who.int/neglected_ diseases/2010report/en/index.html/).

There are dozens of terrible, obscure diseases that could be considered neglected. Choosing 17 for focused action was presumably not easy. Some advocates for other diseases may object that their particular disease of interest was left off the list, whereas others may wonder why some diseases were included. But listing all (or too many) neglected diseases would defeat the purpose, which is to select a limited number of diseases and then do something about them.

The WHO acknowledges that the diseases listed "are not a natural or a uniform group of disorders" and "differ significantly in their causes, pathologies, and clinical features." The diseases also differ in their vulnerability to elimination or control. For example, one of the 17, Guinea worm (dracunculiasis), is on the verge of eradication, whereas considerable progress is being made against onchocerciasis, lymphatic filariasis, leprosy and trachoma, for example.

It's clear that the criteria for choosing 17 NTDs from among the many potential choices included both the global extent of each disease and the existence of tools to control or eliminate it. Now that the careful selection process is complete, it's important to emphasize the difference that effective leadership can make. Compare the examples of two of the 17 NTDs targeted by the WHO: human African trypanosomiasis, also known as sleeping sickness, and yaws, a chronic, debilitating skin and bone disorder caused by the bacterium Treponema pertenue. Despite the severe handicaps of difficult diagnosis, complex treatment and occurrence in violence-prone areas, the reported incidence of human African trypanosomiasis has been halved in the past decade, thanks in part to political will. Meanwhile, even though an inexpensive single injection of long-acting penicillin can cure yaws, which was targeted for eradication over 50 years ago, inadequate attention by all concerned means that this debilitating disease is still mutilating people in several countries.

In its October report, the WHO lists more than 60 resolutions that the World Health Assembly has adopted over the years related to the 17 NTDs, including eleven resolutions for leprosy alone. Guinea worm is the only one of these diseases that is formally targeted by the WHO for global eradication. Seven NTDs are targeted for regional elimination, whereas the other diseases are targeted for better control. Notably, the lack of quantitative measures to specify what is meant by improved control or by "elimination as a public health problem" for many of these represents a fundamental flaw.

Despite these ambiguities, we are now obligated to define measurable goals and monitor progress toward those goals for all of the 17 NTDs selected by the WHO. Additionally, to know whether meaningful progress is being made, we need regular reports of the proportion of the at-risk population that is receiving the appropriate interventions, as well as data on trends in disease prevalence. Relying only on numbers of treatments provided, for example, is not enough. If the goal is to reduce occurrence of a disease to zero by a certain date, as in an eradication or elimination program, progress toward that can be measured. An aim to reduce a disease to less than one case per 10,000 population by a certain date can also be measured. But it is impossible to know whether one has achieved "elimination as a public health problem" without including a quantitative threshold to define success.

When it comes to deciding the best options to fight NTDs, it's important to emphasize the role of personal risk factors in exposure to many of these 17 diseases. Proper attention should also be paid to research for improving health education and understanding sociological aspects of NTDs, as well as behavioral aspects of their prevention in affected communities. For example, the campaign to eradicate Guinea worm disease shows that it is possible to defeat a neglected tropical disease even without a vaccine or a curative treatment. Meanwhile, for those illnesses that do require drug interventions, it is necessary to foster ongoing communication between public health operations and laboratory researchers for the purpose of setting research priorities for translational medicine.

Over the past two decades, tropical diseases have received increased notice, encouraged by unprecedented donations of powerful antibiotics by pharmaceutical companies and enormous financial support by the Bill \& Melinda Gates Foundation, for example. We must not squander this increased attention by failing to discipline ourselves and measure the impact of our work in relation to quantitative goals. The WHO, its partners and the respective countries concerned should establish baseline measurements, set quantified targets for 2015 and 2020, monitor interventions and cases and publish the results annually in a combined summary report as well as more detailed annual surveillance summaries for each disease in the WHO's Weekly Epidemiological Record. The Carter Center's experience is that annual program reviews-in which country representatives present progress reports and interact with their peersencourage action by countries, promote accountability and facilitate reporting.

In releasing its NTD plan, the WHO assumes its appropriate role as chief advocate for improving the health of impoverished people. For now, the organization should resist pressures to continue expanding its current list of 17 NTDs. As some of these diseases are eliminated or controlled, with the hard numbers to back up progress, new diseases might then replace others removed from the list.

Donald R. Hopkins is vice president for Health Programs at the Carter Center in Atlanta, Georgia. 